\title{
Revisión de modelos teóricos de la dinámica de fluidos asociada al flujo de sangre
}

A review of theoretical blood flow models

Gabriela Ortiz-León'

Daniel Araya-Luna²

Marta Vílchez-Monge ${ }^{3}$

Ortiz-León, G; Araya-Luna, D; Vîlchez-

Monge, M. Revisión de modelos teóricos de la dinámica de fluidos asociada al flujo de sangre. Tecnología en Marcha. Vol. 27, N I. Pág 66-76 


\section{Palabras clave}

Modelo de sangre; fluido no newtoniano.

\section{Resumen}

La sangre es un fluido incompresible, viscoelástico y de comportamiento no newtoniano. Su representación pormedio de un modelo matemático debe contemplar estas características, así como el compromiso entre la exactitud de los resultados y el cálculo computacional para obtenerlos. A continuación se presenta un resumen de los modelos de flujo no newtoniano utilizados con mayor frecuencia en la descripción del flujo sanguíneo, una evaluación realizada sobre modelos de sangre seleccionados de la literatura, la recapitulación de los parámetros utilizados para la descripción de la sangre y los resultados de una prueba de concepto para la comparación de modelos de sangre en una arteria.

\section{Key words}

Blood models; Non-Newtonian fluid.

\section{Introducción}

El alto índice de enfermedades cardiovasculares en países desarrollados y en vías de desarrollo ha estimulado la investigación del comportamiento de la sangre en los grandes vasos sanguíneos, con la finalidad de comprender diversas patología cardiovasculares, mejorar los métodos de diagnóstico asociados y reducir costos en el diseño e implementación de dispositivos implantables que tendrían contacto con la sangre, como por ejemplo, válvulas cardíacas y bombas de sangre.

La descripción matemática del comportamiento de la sangre permite estudiar sistemáticamente el comportamiento de los patrones de flujo al variar las características elásticas y geometrías de los vasos sanguíneos, sin exponer a un ser vivo a situaciones experimentales. Por otra parte, el análisis numérico de la dinámica de fluidos permite predecir daños en la sangre debido, por ejemplo, a la implantación de dispositivos médicos.

La sangre es un fluido multifase, dada la presencia de sólidos suspendidos en el plasma, además su viscosidad y densidad son modificadas por factores tales como la temperatura y el hematocrito. Se ha demostrado que es posible modelar la sangre como un fluido homogéneo en los grandes vasos sanguíneos, en los cuales el diámetro es al menos dos órdenes de magnitud mayor que el tamaño de los glóbulos rojos. En este artículo se resume una evaluación de antecedentes y algunos parámetros utilizados por diversos autores en el modelado del flujo sanguíneo.

\section{Procedimiento metodológico}

Lametodología seguidapara esta revisión fueseleccionar de la literatura los modelos de sangre que describen su comportamiento en los vasos sanguíneos; además se definieron variables comparativas para evaluar cualitativamente estos modelos. Posteriormente se resumieron los parámetros utilizados en la descripción matemática del flujo sanguíneo y se realizó una prueba de concepto por medio del método de elementos finitos.

La revisión bibliográfica se enfoca en las descripciones del flujo sanguíneo que puedan ser implementadas 
mediante la dinámica computacional de fluidos (CFD, por sus siglas en inglés). El primer paso fue seleccionar nueve modelos y determinar las variables significativas para la descripción de la sangre a través de las ecuaciones de Navier-Stokes en tres dimensiones para un fluido incompresible, viscoso y no turbulento.

La siguiente etapa fue la evaluación de modelos existentes para determinar las ventajas y desventajas de los modelos seleccionados de la literatura. Para realizar esta actividad se declararon variables cualitativas con el fin de medir el potencial de un modelo matemático determinado.

Finalmente, se construyó una lista de los parámetros utilizados por otros autores para modelar el fluido sanguíneo, entre los que se encuentran la densidad, la viscosidad, el tipo de fluido, la frecuencia cardíaca y la presión arterial. Los modelos newtonianos se compararon con los modelos no newtonianos para determinar el compromiso entre la utilización de recursos computacionales en la simulación y los resultados obtenidos.

\section{Modelos de sangre no newtonianos}

Los efectos no newtonianos de la sangre son despreciados cuando el diámetro del vaso sanguíneo considerado es mucho mayor que el diámetro de las partículas sólidas en el fluido sanguíneo, por ejemplo, en la aorta ascendente (y la aorta torácica en general) y particularmente a velocidades de corte menores a $100 \mathrm{~s}^{-1}$ (Johnston, Johnston, Corney y Kilpatrick, 2004, Finocchiaro et al., 2009). En caso de realizar un estudio sobre la circulación en las redes capilares del sistema circulatorio, la hipótesis de un comportamiento newtoniano pierde validez ya que el diámetro de estos vasos se reduce considerablemente hasta llegar al rango de micrómetros, en otras palabras, conforme se realizan estudios o modelos en vasos sanguíneos distales al corazón, el comportamiento reológico no newtoniano en la interacción fluido-estructura cobra mayor validez. Un modelo de sangre no newtoniano ideal debe satisfacer los siguientes parámetros (Goubergrits, Wellnhofer y Kertzscher, 2008):

- Simular el fenómeno de adelgazamiento por corte ("shear thinning"), es decir, la disminución en la viscosidad cuando se incrementa la tasa tensión de corte.
- Incluir la dependencia del hematocrito.

- Considerar la dependencia de la temperatura.

- Contener la concentración de proteínas en la sangre.

- Valorar las condiciones del flujo, es decir, si el flujo es pulsátil o continuo.

A continuación, se presenta un resumen de los principales modelos no newtonianos utilizados en la descripción del flujo sanguíneo.

\section{Ley de Potencias ("Power Law”)}

Este modelo es una modificación del Modelo Newtoniano con la velocidad de deformación $(\dot{\gamma}$ ) elevada a una potencia que incluye un índice no newtoniano (Goubergrits et al., 2008, Johnston et al., 2004). Este comportamiento es la base para los otros modelos no newtonianos y se expresa por medio de la ecuación I:

$\mu=\mu_{0}(\dot{\gamma})^{n-1}$

La ecuación I representa el cambio en la viscosidad $\mu$ debido a los parámetros $m_{0}$ y $n$, donde $m_{0}$ es un índice de consistencia de valor 0,035 y $n$ es el índice no newtoniano con valor de 0,6. Los parámetros $m_{0}$ y $n$ corresponden a constantes de ajuste determinadas experimentalmente, entre más alto sea $m_{0}$ más viscoso es el fluido y entre más alejado se encuentre $n$ de la unidad, las características no newtonianas se hacen más pronunciadas (Kim, 2002).

\section{Modelo Casson}

Este modelo es una modificación de la Ley de Potencias que incluye la dependencia de la viscosidad con el hematocrito (Goubergrits et al., 2008). La expresión de la viscosidad en este caso se considera como:

$$
\mu=\left(\sqrt{\mu_{\infty}}+\sqrt{\frac{\lambda_{y}}{\dot{\gamma}}}\right)^{2}
$$

Los parámetros utilizados son: $\mathrm{m}_{0}=0,0012,0<$ $H<\mathrm{I}$, donde $\mathrm{H}$ representa el hematocrito, es decir, el porcentaje de eritrocitos en la sangre, $\lambda_{y}=0,0 \mathrm{I}(0,625 \mathrm{H})^{3}$ y $\mu_{\infty}=\mu_{0} *(\mathrm{I}-\mathrm{H})^{-2,5}$. Ambas dependencias al hematocrito $\left(\lambda_{y}\right.$ y $\left.\mu_{\infty}\right)$ son ajustadas experimentalmente (Goubergrits et al., 2008). 


\section{Modelo Carreau}

Este modelo asume que la viscosidad varía siguiendo la siguiente ecuación (Shibeshi \& Collins, 2006):

$$
\mu=\mu_{\infty}+\left(\mu_{0}-\mu_{\infty}\right)\left[1+(\lambda \dot{\gamma})^{2}\right]^{\frac{n-1}{2}}
$$

En este modelo $\mu_{0}$ y $\mu_{\infty}$ son las viscosidades límites a razones de deformación por presión de cero e infinito respectivamente, $\lambda$ es la constante de relajación y $n$ es el índice No-Newtoniano del modelo de Ley de Potencias. Los valores experimentales recomendados para este modelo son: $\mu_{\infty}=0,00345$ $\mathrm{Ns} / \mathrm{m}^{2}, \mu_{0}=0,056 \mathrm{Ns} / \mathrm{m}^{2}, \lambda=10,976$ y $\mathrm{n}=0,3568$ (Johnston et al., 2004).

\section{Modelo Walburn-Schneck}

Este modelo no newtoniano es también una modificación del modelo de la Ley de Potencias. Walburn y Schneck describieron los datos experimentales obtenidos de sangre con anticoagulantes por medio de cuatro constantes y dos parámetros que incluyen la dependencia al hematocrito $(H)$ y la concentración total de proteína menos albúmina (TPMA) (Goubergrits et al., 2008). La viscosidad se describe con la siguiente expresión:

$$
\mu=C_{1} e^{C_{2} H}\left[e^{C_{4}\left(\frac{T P M A}{H^{2}}\right)}\right](\dot{\gamma})^{-C_{3} H}
$$

donde las constantes se determinaron experimentalmente en: $C_{1}=0,00797 ; C_{2}=0,0608 ; C_{3}=0,00499 \mathrm{y}$ $C_{4}=14,585$. A partir de los resultados experimentales que obtuvieron, estos autores proponen un modelo con $H=40 \%$ y $T P M A=25,9 \mathrm{~g} / \mathrm{l}$.

\section{Modelo GPL ("Generalized Power Law”)}

En este estudio se incorpora el modelo de la Ley de Potencias a bajas velocidades de corte, el modelo newtoniano a velocidades de corte media y altas $y$, en consecuencia, el Modelo Casson se considera un caso límite. La viscosidad en este caso se describe como:

$$
\mu=0,1 \lambda(\dot{\gamma})^{n-1}
$$

donde

$$
\begin{aligned}
& \lambda(\dot{\gamma})=\mu_{\infty}+\Delta \mu e^{\left[-\left(1+\frac{\dot{\gamma}}{a}\right) e^{-b / \hat{\gamma}}\right]} \\
& n(\dot{\gamma})=\mu_{\infty}+\Delta n e^{\left[-\left(1+\frac{\dot{\gamma}}{c}\right) e^{-d / \dot{\gamma}}\right]}
\end{aligned}
$$

Este modelo tiene los siguientes valores ajustados experimentalmente: $\mu_{\infty}=0,035 ; n_{\infty}=1,0 ; \quad \Delta \mu=0,25$; $\Delta n=0,45 ; \mathrm{a}=50 ; \mathrm{b}=3 ; \mathrm{c}=50$ y $\mathrm{d}=4$.

\section{Modelo GPL modificado}

En este caso se modifica el Modelo GPL para incluir la dependencia con el hematocrito, la temperatura y la concentración de TPMA (Goubergrits et al., 2008), (Johnston et al., 2004). La expresión de la viscosidad está dada por:

$\left.\mu=C_{2} e^{\left(\frac{C_{1} T P M A}{H^{2}}\right)} \cdot\left[\mu_{0}(1-H)^{-2,5}+\Delta \mu e^{\left[-\left(1+\frac{\dot{\gamma}}{A} e^{-B / \gamma}\right.\right.}\right]\right]$

Donde

$A=a_{1} H^{2}+a_{2} H+a_{3} T+a_{4}$

$B=b_{1} H^{2}+b_{2} H+b_{3} T+b_{4}$

Con los siguientes valores ajustados experimentalmente: $\quad \mu_{0}=0,0008585 ; \quad \Delta \mu=0,00707$; $C_{1}=0,0019225 ; C_{2}=0,7639 ; 0<H<1 ; a_{1}=0,14054375$; $a_{2}=-14,7089379 ; a_{3}=1,3072387 ; a_{4}=383,039 ; b_{1}=$ 0,$02162045 ; \quad b_{2}=2,1138407 ; \quad b_{3}=-0,193464 ; \quad b_{4}=-$ 41,2627

El modelo GPL modificado presenta las mismas ventajas que el modelo GPL e incorpora la concentración de TPMA, lo que significa que se obtiene un modelo más flexible, con la consecuencia de que, al tener una mayor cantidad de coeficientes en la ecuación constitutiva, aumenta la demanda en el procesamiento computacional de las simulaciones.

\section{Evaluación cualitativa de antecedentes}

En las simulaciones de la interacción fluido-estructura entre el flujo sanguíneo y las arterias de mayor diámetro, como el caso de la aorta, es común el uso de fluidos newtonianos. En este caso se asume que la sangre se comporta como un fluido homogéneo y 
con viscosidad constante, también se asume que la velocidad de corte es mayor a $100 \mathrm{~s}^{-1}$ y el diámetro de los vasos es mucho mayor al tamaño de los glóbulos rojos.

Con el objetivo de evaluar los antecedentes y simulaciones realizadas por diferentes autores en la literatura disponible, se presenta la definición de un grupo de indicadores utilizados para analizar las características de los modelos estudiados, así como la descripción de la escala de medición que se usó para calificar cada una de las variables definidas:

I. Condiciones de entrada/salida: Descripción de las condiciones que se aplicaron a la entrada y/o salida del modelo; se consideran entradas de presión y velocidad y si el flujo es constante o pulsátil.

2. Flexibilidad: Grado en que la simulación permite introducir modificaciones en sus parámetros. Si el modelo no indica o no permite modificaciones en la evaluación, se indica con el símbolo (×).

3. Tipo de pared: Descripción de las condiciones que se utilizaron para modelar la pared arterial; si el modelo no indica el tipo de material se indica con el símbolo $(\times)$.

4. Interacción fluido-estructura: Grado en que se desarrolló la interacción entre la pared arterial y el fluido sanguíneo. Se definen las siguientes escalas:

- Acople fuerte: Interacción en ambos sentidos fluido-estructura $(\sqrt{ })$.

- Acople débil: Interacción en un solo sentido de fluido a estructura $(\leftrightarrow)$.

- No incluye interacción ( $\times$ ).

5. Consumo de recursos computacionales: Capacidad de procesamiento requerida para la resolución del modelo. En la mayoría de los estudios consultados no se establece de forma explícita el consumo de recursos computacionales, por lo que se realizó una estimación de acuerdo con las dimensiones (2D y 3D) y la cantidad de elementos utilizados para la resolución del modelo. La escala utilizada se describe a continuación:

- Básica: La simulación puede llevarse a cobo en una PC con core DUO y 4 GB de RAM o menos.
- Media: Requiere una cantidad de memoria RAM mayor a 4 GB.

- Alta: Requiere el uso de un Cuad-core y hasta 8 GB de memoria RAM.

- Muy alta: Se requiere más de un Cuad-core.

6. Precisión: Grado en que los resultados obtenidos se parecen a los valores típicos o normales. Este indicador se califica como:

- Satisfactoria $(\sqrt{ })$.

- Parcialmente satisfactoria $(\leftrightarrow)$.

- Deficiente (x).7.Pertinencia de la documentación: Grado en que la documentación existente describe el funcionamiento de la simulación.

8. Acople entre diferentes materiales: grado en que se incluyen diferentes clases de materiales al modelo.

Los resultados de la evaluación de los modelos estudiados se muestran en el cuadro I. En esta tabla se observa que en los modelos estudiados no se incluye el acople entre materiales con características diferentes, como sería el caso del acople de un dispositivo implantable y la arteria. También se destaca que en las publicaciones evaluadas se considera la interacción del flujo sobre la estructura, pero ningún caso contempla el efecto de la deformación de la estructura sobre el nuevo patrón de flujo, es decir, los modelos no involucran la interacción fluidoestructura en ambos sentidos.

Se observa que los principales parámetros en estos modelos son la densidad y la viscosidad dinámica. Para un modelo de sangre newtoniano, incompresible y homogéneo, los valores normales de estos parámetros varían en la literatura entre $1044 \mathrm{~kg} /$ $\mathrm{m}^{3}$ y $1176 \mathrm{~kg} / \mathrm{m}^{3}$ para la densidad y entre $3.05 \mathrm{mPa}$ s y $3.5 \mathrm{mPa}$ s para la viscosidad (Tan et al., 2008; Watson, 2007, Mabotuwana et al., 2007, Ubal et al., 2010).

\section{Parámetros para modelado de fluido sanguíneo}

Las características y composición propias de la sangre hacen difícil la construcción de un modelo funcional que pueda ser utilizado en diversos estudios, por lo cual es común realizar simplificaciones para cada caso de estudio particular. La literatura consultada (cuadro I) presenta diversas aproximaciones a 
Cuadro I. Evaluación cualitativa de antecedentes consultados.

\begin{tabular}{|c|c|c|c|c|c|c|c|c|}
\hline Ref & $\begin{array}{c}\text { Condición } \\
\text { entrada-salida }\end{array}$ & Flexibilidad & $\begin{array}{l}\text { Tipo de } \\
\text { pared }\end{array}$ & $\begin{array}{l}\text { Interacción } \\
\text { fluido- } \\
\text { estructura }\end{array}$ & $\begin{array}{l}\text { Consumo } \\
\text { de recursos }\end{array}$ & Precisión & $\begin{array}{l}\text { Docu- } \\
\text { men- } \\
\text { tación }\end{array}$ & $\begin{array}{c}\text { Acople } \\
\text { entre } \\
\text { materiales }\end{array}$ \\
\hline $\begin{array}{l}\text { (Tan et al., } \\
\text { 2008) }\end{array}$ & $\begin{array}{c}\text { Velocidad } \\
\text { pulsátil tres } \\
\text { ciclos cardíacos } \\
\text { e intensidad de } \\
\text { turbulencia I,5\% } \\
\text { a la entrada. } \\
\text { A la salida } \\
\text { 40-60\% del flujo } \\
\text { de entrada }\end{array}$ & $x$ & $x$ & $x$ & Media & $\sqrt{ }$ & $x$ & $\times$ \\
\hline $\begin{array}{l}\text { Watson, } \\
\text { 2007) }\end{array}$ & $\begin{array}{l}\text { Onda de presión } \\
\text { para un ciclo } \\
\text { cardíaco a la } \\
\text { entrada. } \\
\text { Condiciones de } \\
\text { desplazamiento } \\
\text { en la pared } \\
\text { ventricular. }\end{array}$ & $\begin{array}{l}\text { Parámetros } \mathrm{R} \\
\text { y } \mathrm{C} \text { permiten } \\
\text { modelar arterias } \\
\text { rígidas y elásticas } \\
\text { respectivamente }\end{array}$ & $\begin{array}{l}\text { Rígida y/o } \\
\text { flexible }\end{array}$ & $\leftrightarrow$ & Media & $\leftrightarrow$ & $\sqrt{ }$ & $x$ \\
\hline $\begin{array}{c}\text { (Mabotuwana, } \\
\text { Cheng y } \\
\text { Pullan, 2007) }\end{array}$ & $\begin{array}{l}\text { Onda de presión } \\
\text { en la entrada } \\
\text { para dos ciclos } \\
\text { cardíacos y } \\
\text { relación presión- } \\
\text { radio en el } \\
\text { interior de la } \\
\text { geometría }\end{array}$ & $\begin{array}{l}\text { La variaciónes } \\
\text { en el radios del } \\
\text { conducto. } \\
\text { Densidad, } \\
\text { viscosidad } \\
\text { y perfil de } \\
\text { velocidad. }\end{array}$ & Elástica & $x$ & Alta & $\sqrt{ }$ & $\sqrt{ }$ & $\times$ \\
\hline $\begin{array}{c}\text { (Ubal, } \\
\text { Campana, } \\
\text { Rodríguez, } \\
\text { Berli y Paolo, } \\
\text { 20 I0) }\end{array}$ & $\begin{array}{c}\text { Variación } \\
\text { senoidal del } \\
\text { caudal para tres } \\
\text { semiciclos en la } \\
\text { entrada }\end{array}$ & $\begin{array}{l}\text { Densidad y } \\
\text { viscosidad } \\
\text { sanguínea }\end{array}$ & Rígida & $x$ & Media & $x$ & $\sqrt{ }$ & $x$ \\
\hline $\begin{array}{l}\text { (Leuprecht, } \\
\text { Kozerke, } \\
\text { Boesiger y } \\
\text { Perktold, } \\
\text { 2003) }\end{array}$ & $\begin{array}{c}\text { Perfil de } \\
\text { velocidad tipo } \\
\text { Plug-In a la } \\
\text { entrada y perfil } \\
\text { de deformación } \\
\text { aplicado a } \\
\text { las paredes } \\
\text { obtenidos } \\
\text { mediante MRI }\end{array}$ & $\begin{array}{l}\text { Densidad y } \\
\text { viscosidad } \\
\text { sanguínea }\end{array}$ & $x$ & $x$ & Alta & $\sqrt{ }$ & $\sqrt{ }$ & $x$ \\
\hline $\begin{array}{l}\text { (Goubergrits } \\
\text { et al., 2008) }\end{array}$ & $\begin{array}{c}\text { Perfil de } \\
\text { velocidad en la } \\
\text { entrada pero no } \\
\text { se indica si es } \\
\text { pulsátil. } \\
\text { Razón de flujo } \\
\text { aplicado a la } \\
\text { salida }\end{array}$ & $\begin{array}{c}\text { Modelos } \\
\text { paramétricos, } \\
\text { permiten ajustes } \\
\text { con datos } \\
\text { experimentales }\end{array}$ & Rígida & $x$ & Alta & $\sqrt{ }$ & $\sqrt{ }$ & $x$ \\
\hline
\end{tabular}




\section{Continuación}

\begin{tabular}{|c|c|c|c|c|c|c|c|c|}
\hline Ref & $\begin{array}{c}\text { Condición } \\
\text { entrada-salida }\end{array}$ & Flexibilidad & $\begin{array}{l}\text { Tipo de } \\
\text { pared }\end{array}$ & $\begin{array}{l}\text { Interacción } \\
\text { fluido- } \\
\text { estructura }\end{array}$ & $\begin{array}{l}\text { Consumo } \\
\text { de recursos }\end{array}$ & Precisión & $\begin{array}{l}\text { Docu- } \\
\text { men- } \\
\text { tación }\end{array}$ & $\begin{array}{c}\text { Acople } \\
\text { entre } \\
\text { materiales }\end{array}$ \\
\hline $\begin{array}{c}\text { (Gao, } \\
\text { Watanabe y } \\
\text { Matsuzawa, } \\
\text { 2006) }\end{array}$ & $\begin{array}{c}\text { Perfil de } \\
\text { velocidad pulsátil } \\
\text { a la entrada y } \\
\text { presión cero } \\
\text { aplicado a la } \\
\text { salida }\end{array}$ & $x$ & Elástica & $\sqrt{ }$ & Alta & $\leftrightarrow$ & $\sqrt{ }$ & $x$ \\
\hline $\begin{array}{l}\text { (Vasava, Jalali y } \\
\text { Dabagh, 2009) }\end{array}$ & $\begin{array}{l}\text { Condiciones de } \\
\text { presión aplicadas } \\
\text { tanto a la entrada } \\
\text { como a la salida }\end{array}$ & $\begin{array}{c}\text { Densidad y } \\
\text { viscosidad } \\
\text { sanguínea }\end{array}$ & Rígida & $x$ & Alta & $\sqrt{ }$ & $\sqrt{ }$ & $x$ \\
\hline $\begin{array}{l}\text { (Gao, Guo, } \\
\text { Sakamoto y } \\
\text { Matsuzawa, } \\
\text { 2006) }\end{array}$ & $\begin{array}{c}\text { Perfil de } \\
\text { velocidad a la } \\
\text { entrada. No } \\
\text { se indican las } \\
\text { condiciones } \\
\text { aplicadas a la } \\
\text { salida }\end{array}$ & $x$ & Elástica & $\sqrt{ }$ & Alta & $\leftrightarrow$ & $x$ & $x$ \\
\hline
\end{tabular}

esta labor. Los parámetros utilizados por diferentes autores para modelar el fluido sanguíneo se presentan en el cuadro 2, que incluye características como tipo de fluido, densidad, viscosidad y, por último, particularidades del flujo utilizado para el modelo respectivo (Araya-Luna, 20I2).

Se observa que los principales parámetros en estos modelos son la densidad y la viscosidad dinámica. Para un modelo de sangre newtoniano, incompresible y homogéneo, los valores normales de estos parámetros varían en la literatura entre $1044 \mathrm{~kg} / \mathrm{m}^{3}$ y $1176 \mathrm{~kg} / \mathrm{m}^{3}$ para la densidad y entre $3,05 \mathrm{mPa}$ s y 3,5 mPa s (Zakaria, Robertson y Kerber, 2008).

\section{Análisis comparativo entre modelos newtonianos y no newtonianos}

Con la finalidad de observar las diferencias en el comportamiento del flujo con diferentes modelos y validar las aproximaciones implementadas, se contrastaron los resultados de tres modelos de flujo de sangre diferentes: un modelo newtoniano y los modelos no newtonianos de Ley de Potencias y Carreau.

El modelo sanguíneo desarrollado se basa en las ecuaciones de Navier-Stokes para un fluido viscoso e incompresible. El segmento de arteria utilizado para las simulaciones, por su composición y características mecánicas, se considera como un material viscoelástico con un Módulo de Young (E) de 2,5 $\mathrm{MPa}$, una densidad de tejido $(\rho)$ de $960 \mathrm{~kg} / \mathrm{m}^{3}$ y una razón de Poisson (v) de 0,5 (Gao, Guo, et al., 2006; Gao, Watanabe, et al., 2006). La geometría empleada está formada por una tubería cilíndrica con longitud de $150 \mathrm{~mm}$, un radio externo de 25 $\mathrm{mm}$ y un grosor de pared arterial de $2 \mathrm{~mm}$. Los modelos se resolvieron mediante la plataforma de simulación COMSOL Multiphysics ${ }^{\circledR}$ usando métodos de elementos finitos para un cuarto de la geometría y aplicando condiciones de simetría, como se muestra en la figura I.

Las condiciones de frontera aplicadas tienen la forma de onda de presión característica en la salida de la válvula aórtica. En la figura 2 se muestran la presión en la entrada y en la salida. La condición de frontera aplicada a la salida es la onda de entrada atenuada y desfasada; la atenuación se debe al gasto enérgetico por efectos de transporte y con el desfase se considera la velocidad de propagación de onda. La atenuación de la presión promedio es de 34 Pa (Trainini, 2004), lo que equivale a una caída de presión de 2 Palcm (Anza \& Esteves, 20 I I). Por otro lado, la teoría de propagación de ondas aplicada a la biomécanica indica que la velocidad de propagación 
Cuadro 2. Parámetros establecidos en modelos de fluido sanguíneo.

\begin{tabular}{|c|c|c|c|}
\hline Tipo de fluido & $\begin{array}{l}\text { Densidad } \\
(\mathrm{r})\left[\mathrm{kg} / \mathrm{m}^{3}\right]\end{array}$ & Viscosidad (m) [mPa s] & Características del flujo \\
\hline $\begin{array}{l}\text { Newtoniano incompresible } \\
\text { (Tan et al., 2008) }\end{array}$ & 1176 & 3,34 & $\begin{array}{l}\text { Pulsátil (paso 0,0। s) } \\
\text { Razón de flujo de salida } 40-60 \% \text { del flujo de } \\
\text { entrada }\end{array}$ \\
\hline $\begin{array}{l}\text { Newtoniano } \\
\text { incompresible(Ghista, Zhong, } \\
\text { Ng, \& Tan, n.d.) }\end{array}$ & - & - & $\begin{array}{c}p=100 \mathrm{mmHg} ; \mathrm{Dp}=20 \mathrm{mmHg} \text { (Normal) } \\
p=120 \mathrm{mmHg} ; \mathrm{D}=20 \mathrm{mmHg} \text { (aterosclerosis) } \\
p=100 \mathrm{mmHg} ; \mathrm{Dp}=30 \mathrm{mmHg} \text { (arterosclerosis) }\end{array}$ \\
\hline $\begin{array}{l}\text { Newtoniano y No } \\
\text { Newtoniano (Generalized } \\
\text { Power Law) (Watson, 2007) }\end{array}$ & 1060 & 2,7 & $\begin{array}{c}\text { Geometría del corazón en 2D, utiliza flujo } \\
\text { laminar }\end{array}$ \\
\hline $\begin{array}{l}\text { Newtoniano incompresible, } \\
\text { homogéneo (Mabotuwana et } \\
\text { al., 2007) }\end{array}$ & 1050 & $3,2\left(\mathrm{~cm}^{2} / \mathrm{s}\right)$ & $\begin{array}{c}\text { Flujo axisimétrico } \\
\mathrm{P}_{0}=12,4 ; \mathrm{Dp}=2,2 \mathrm{kPa} \\
\mathrm{P}_{\text {out }}=\text { cte }=11,0 \mathrm{kPa}\end{array}$ \\
\hline $\begin{array}{l}\text { Newtoniano incompresible } \\
\text { (Ubal et al., 2010) }\end{array}$ & 1050 & 3,05 & $F_{c}=70-180 \mathrm{lat} / \mathrm{min}$ \\
\hline $\begin{array}{l}\text { Newtoniano incompresible } \\
\text { (Zhao-rong, Gang, Yong, } \\
\text { Zhong-zhao, \& Kai-rong, 2003) }\end{array}$ & 1050 & 4,0 & $\begin{array}{l}\text { Flujo estacionario bajo presión promedio } \\
\mathrm{P}_{\mathrm{m}}=13,5 \mathrm{kPa} \text { combinado con flujo pulsátil }\end{array}$ \\
\hline $\begin{array}{l}\text { Newtoniano incompresible } \\
\text { (Zakaria et al., 2008) }\end{array}$ & 1050 & 3,5 & $\mathrm{f}_{0}=1 \mathrm{~Hz} 60 \mathrm{lat} / \mathrm{min}$ \\
\hline $\begin{array}{l}\text { Newtoniano 3D incompresible } \\
\text { (Leuprecht et al., 2003) }\end{array}$ & 1044 & 3,65 & $\begin{array}{l}\omega=7,34 \mathrm{~s}^{-1} \text { corresponde a } 70 \mathrm{lat} / \mathrm{min} \\
\text { y velocidad media de entrada } v_{0}=1,32 \times 10^{-1} \mathrm{~m} / \mathrm{s}\end{array}$ \\
\hline $\begin{array}{l}\text { No newtoniano (Goubergrits } \\
\text { et al., 2008) }\end{array}$ & - & $\begin{array}{l}\text { Modelo de Casson modificado } \\
\text { Modelo de Walburn y Schneck } \\
\text { Generalized Power Law (GPL) } \\
\text { GPL modificado }\end{array}$ & $\begin{array}{l}\text { Perfil de velocidad "plug" asumido la entrada. } \\
\text { Una relación de tasa de flujo asumida en las } \\
\text { salidas de la bifurcación de 50/45/5 }\end{array}$ \\
\hline $\begin{array}{l}\text { Incompresible y viscoso (Rayz } \\
\text { \& Berger, 2010) }\end{array}$ & - & - & $\begin{array}{l}\text { Perfil de velocidad parabólico o uniforme } \\
\text { asumido en la entrada } \\
\text { Presión ambiente o preescrito a la salida. }\end{array}$ \\
\hline Newtoniano & 1050 & 3,5 & - \\
\hline $\begin{array}{l}\text { Homogéneo e incompresible } \\
\text { (Vasava et al., 2009) }\end{array}$ & 1060 & 5 & 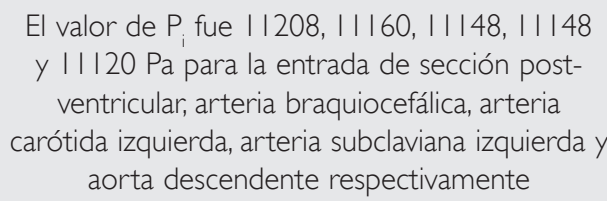 \\
\hline
\end{tabular}

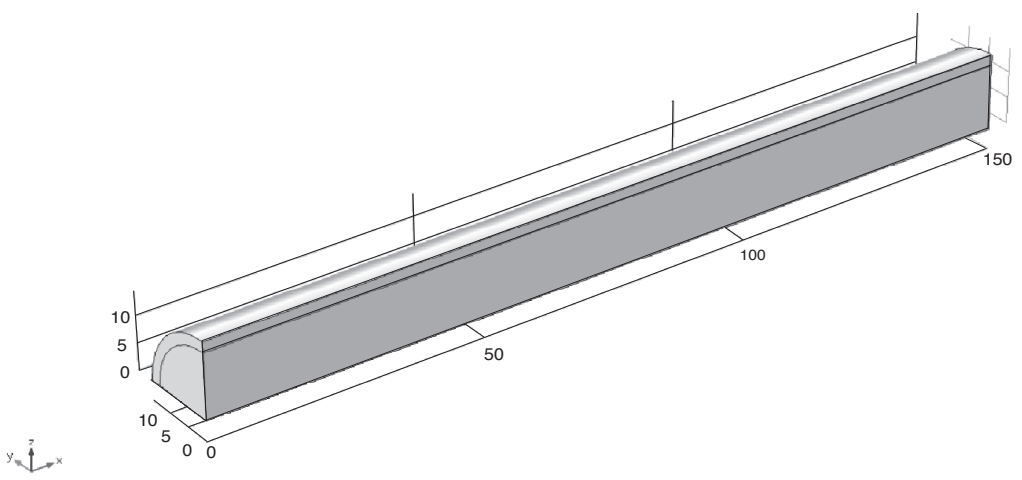

Figura I. Geometría utilizada en la evaluación de modelos de sangre (las escalas están en mm). 


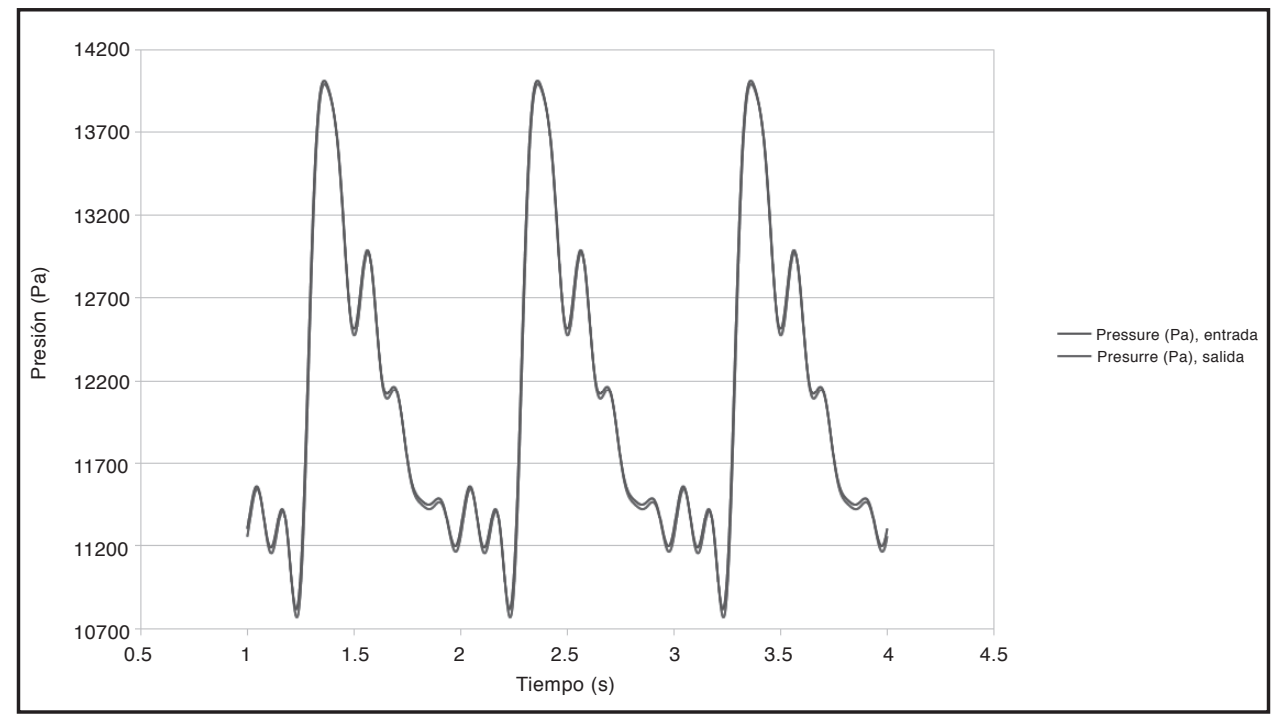

Figura 2: Condiciones de entrada y salida en el conducto arterial utilizadas en las simulaciones.

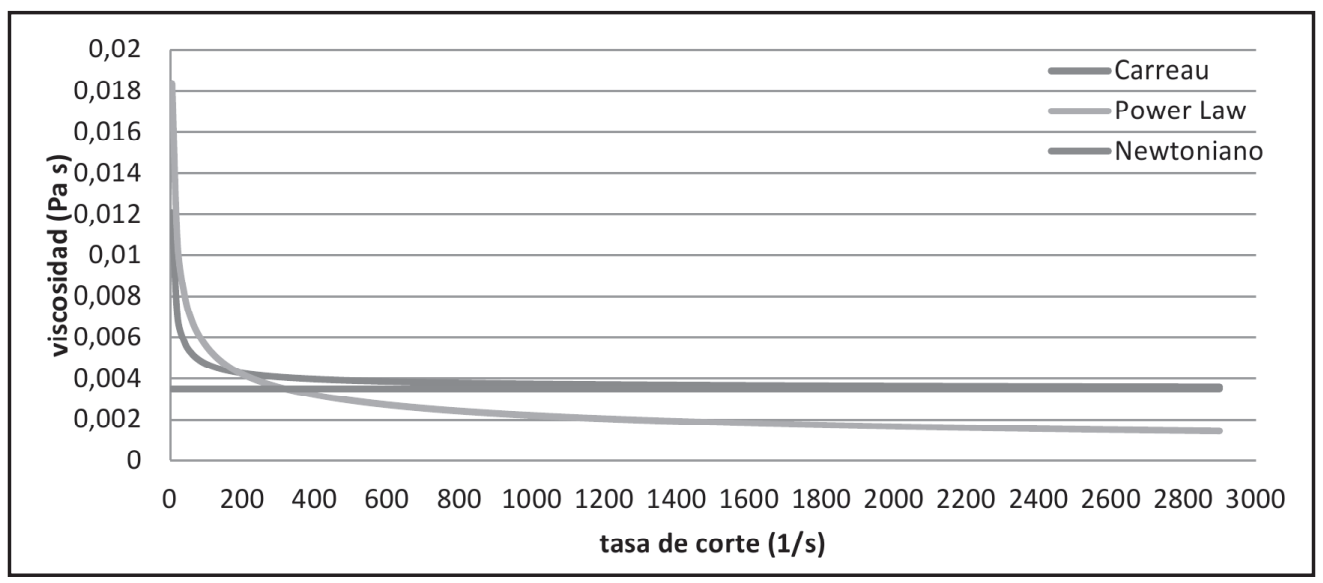

Figura 3. Viscosidad en función de la tasa de corte para tres modelos de sangre (Newtoniano, de Carreau y de Ley de Potencia).

es de aproximadamente $83,3 \mathrm{~cm} / \mathrm{s}$ (Li, 2004). La señal se construye utilizando hasta el octavo armónico de la serie de Fourier de la presión en la aorta ascendente mostrada en (Li, 2004) y el desfase en la salida depende de la longitud de la pared arterial (Araya-Luna, 20I2). El cálculo de los coeficientes de la serie de Fourier se realizó utilizando MATLAB®.

El comportamiento de la viscosidad en función de la tasa de corte para los modelos Newtoniano, de Ley de Potencias y de Carreau se presenta en la figura 3, en la cual se observa que la viscosidad predicha tanto por el Modelo de Carreau como por el de Ley de Potencias aumentan considerablemente para tasas de corte menores a $100 \mathrm{~s}^{-1}$. A altas tasas de corte, superiores a $1600 \mathrm{~s}^{-1}$, se observa que el modelo de Ley de Potencias disminuye hasta un 50\% de los valores típicos esperados; por otro lado, se observa la concordancia que existe entre el Modelo de Carreau y el Modelo newtoniano para un amplio rango de tasas de corte $\left(1000-2900 \mathrm{~s}^{-1}\right)$.

Estos resultados concuerdan con lo esperado a partir del estudio de modelos no newtonianos. La principal ventaja de la Ley de Potencias es su simpleza; requiere únicamente dos parámetros, lo cual significa que los recursos computacionales necesarios para procesar este modelo son bastante 
bajos. No obstante, su principal carencia radica en que no es capaz de predecir de forma adecuada el comportamiento de los fluidos no newtonianos tanto en regiones de altas como de bajas tasas de corte (Kim, 2002). Para el caso del fluido sanguíneo a bajas tasas de corte $\left(200 \mathrm{~s}^{-1}\right)$, la viscosidad aumenta rápidamente, llegando a valores críticos para tasas de corte cercanas a $0 \mathrm{~s}^{-1}$ donde $\mathrm{m}$ tiende a infinito. Por otro lado, en altas tasas de corte $\left(>\mid 200 \mathrm{~s}^{-1}\right)$ este modelo subvalora la viscosidad.

El Modelo de Carreau requiere cuatro parámetros, dos más que el Modelo de Ley de Potencias, pero ofrece un comportamiento más adecuado tanto para altas como para bajas tasas de corte. A altas tasas de corte la viscosidad tiende al valor utilizado para modelos newtonianos 3,5 $\mathrm{mPa}$ s, mientras que a bajas tasas de corte el valor de la viscosidad tiende a $\boldsymbol{\mu}_{0}=0,056$ Pa s. Su principal desventaja es que no incluye los efectos del hematocrito, por lo que la adaptación del modelo a condiciones de un paciente en particular es difícil de lograr.

También se observa que a bajas tasas de corte el comportamiento no newtoniano de la sangre es más pronunciado; esto también concuerda con estudios reológicos de sangre que muestran que a tasas de corte inferiores a $100 \mathrm{~s}^{-1}$ los glóbulos rojos tienden a agruparse (coagulación de la sangre), aumentando así las características no newtonianas del fluido (Behbahani et al., 2009, Finocchiaro et al., 2009, Owens, 2006, Shibeshi y Collins, 2006). Las tasas típicas de corte de la sangre en el cuerpo humano son superiores a $100 \mathrm{~s}^{-1}$. En estudios experimentales (Behbahani et al., 2009) se ha demostrado que a estas tasas de corte, la viscosidad de la sangre humana con un hematocrito de $45 \%$ alcanza valores constantes de entre 3,5 y $4.0 \mathrm{mPa}$.

En la figura 4 se presenta el perfil de la velocidad para los tres modelos, utilizando condiciones iniciales para simular el comportamiento de la sangre con tasas de corte entre 200 y $800 \mathrm{~s}^{-1}$. Se observa que tanto para el Modelo de Carreau como para el Newtoniano la velocidad presenta el mismo orden de magnitud, en tanto que la diferencia del Modelo de Ley de Potencias se debe a que este subestima la viscosidad sanguínea y eso provoca un aumento en la velocidad en comparación con los otros dos modelos. Estos resultados permiten justificar el uso de un modelo newtoniano en la caracterización de un modelo de sangre en vasos sanguíneos mayores.

\section{Conclusión}

Este trabajo describe la dinámica de la sangre en vasos sanguíneos mayores, resumiendo diferentes modelos y parámetros utilizados para la descripción matemática del comportamiento de la sangre. Esta descripciones permiten explotar el potencial de la dinámica computacional de fluidos en el estudio de diversas enfermedades, así como la influencia sobre el comportamiento reológico de la sangre al estar en contacto con elementos implantables. La ventaja de utilizar estas descripciones matemáticas se traduce en menores costos de implementación de experimentos in-vitro e in-vivo, mayor facilidad para estudiar los efectos de cambios en la geometrías de los elementos involucrados y menor infraestructura para realizar estos estudios.

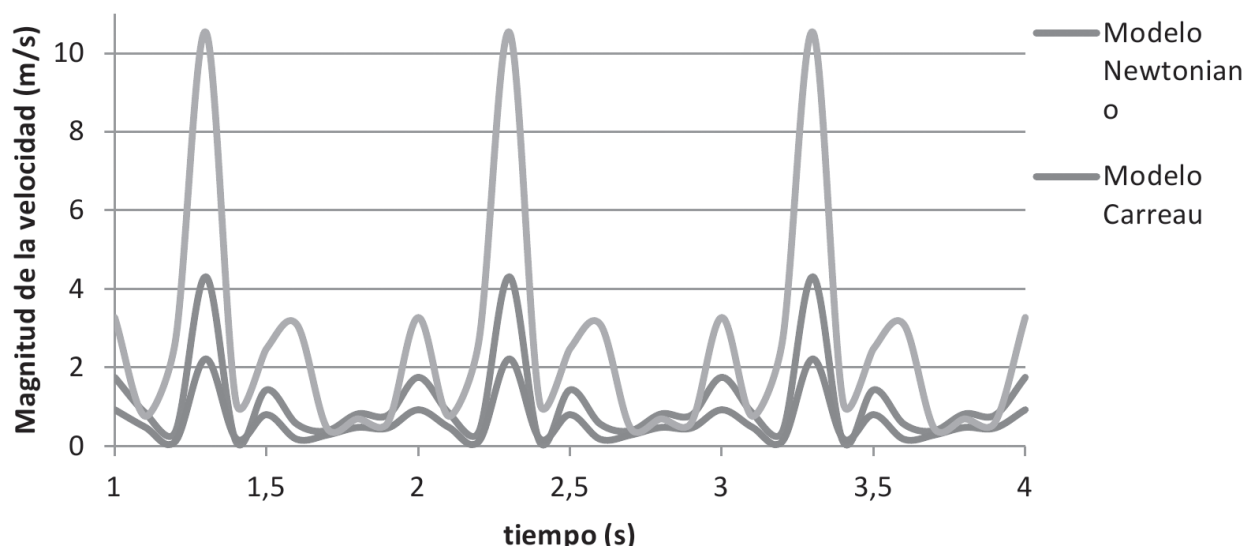

Figura 4. Perfil de velocidad media en función del tiempo para los tres modelos implementados utilizando condiciones iniciales. 


\section{Bibliografía}

Anza, J.J. \& Esteves, M.A. (20I I ). Fluid Structure Interaction Applied to Upper Aorta Blood Flow . 201 I COMSOL Conference in Stuttgart.

Araya-Luna, D.E. (20I2). Modelado de la interacción del fluido sanguíneo sometido a presión pulsátil en la unión de un conducto arterial y un conducto rígido (p. 140).

Behbahani, M., Behr, M., Hormes, M., Steinseifer, U., Arora, D. Coronado, O. \& Pasquali, M. (2009). A review of computational fluid dynamics analysis of blood pumps. European Journal of Applied Mathematics 20(04): 363-397. doi: 10.1017 / S0956792509007839.

Finocchiaro, T., Heinke, S., Leßmann, M., Laumen, M., Steinseifer, U., Schmitz-rode, T., Leonhardt, S. et al. (2009). Design , development, and validation of new VAD / TAH concepts. Biomedizinische Technik/Biomedical Engineering 54: 269-28I. Recuperado desde http://www.cats.rwth-aachen. de:8080/ mb/article-BMT2009a.pdf.

Gao, F., Guo, Z., Sakamoto, M. \& Matsuzawa, T. (2006). Fluidstructure interaction within a layered aortic arch model. Journal of biological physics 32(5): 435-54. doi:10.1007/ sl 0867-006-9027-7.

Gao, F., Watanabe, M. \& Matsuzawa, T. (2006). Stress analysis in a layered aortic arch model under pulsatile blood flow. Biomedical engineering online 5, 25. doi: I0.1 |86/1475925X-5-25.

Ghista, D.N., Zhong, L., Ng, E.Y K. \& Tan, R.S. (n.d.). Arterial Wave Propagation and Reflection at a Bifurcation Site. Pulse (p. 289-310).

Goubergrits, L., Wellnhofer, E. \& Kertzscher, U. (2008). Choice and Impact of a Non-Newtonian Blood Model for Wall Shear Stress Profiling of Coronary Arteries. En: Katashev, A., Dekhtyar, Y., Spigulis, J. \& Magjarevic, R. (Eds.), 14th NordicBaltic Conference on Biomedical Engineering and Medical Physics. IFMBE Proceedings (p. |||-||4). Springer Berlin Heidelberg. doi: I 0.1007/978-3-540-69367-3_30.

Johnston, B.M., Johnston, P.R., Corney, S. \& Kilpatrick, D. (2004). Non-Newtonian blood flow in human right coronary arteries: steady state simulations. Journal of biomechanics 37(5): 709-20. doi: 1 0.1 0 I6/j.jbiomech.2003.09.016.

Kim, S. (2002). A Study of Non-Newtonian Viscosity and Yield Stress Blood in a Scanning Capillary-Tube Rheometer. Drexel University.

Leuprecht, A., Kozerke, S., Boesiger, P. \& Perktold, K. (2003). Blood flow in the human ascending aorta: a combined MRI and
CFD study. Journal of Engineering Mathematics 47(3/4): 387 404. doi: I0. I 023/B:ENGI.0000007969. I 8 I05.b7.

Li, J.K.J. (2004). Dynamics of the Vascular System. Series on Bioengineering, Vol. I. (J.K.-J. Li, Ed.) (World Scie., p. 272).

Mabotuwana, T.D.S., Cheng, L.K. \& Pullan, A.J. (2007). A model of blood flow in the mesenteric arterial system. Biomedical engineering online 6, 17. doi: 10.1 |86/1475-925X-6- 17.

Owens, R.G. (2006). A new microstructure-based constitutive model for human blood. Journal of non-newtonian fluid mechanics I 40(I-3): 57-70. doi: I 0. I O I 6/j.jnnfm.2006.0 I.0 I5

Rayz, V.L. \& Berger, S.A. (2010). Computational Modeling of Vascular Hemodynamics. En: S. De, F. Guilak \& M. Mofrad R.K. (Eds.), Computational Modeling in Biomechanics (p. I7I206). Dordrecht: Springer Netherlands. doi: I0.1007/978$90-48 \mid-3575-2$.

Shibeshi, S. \& Collins, W.E. (2006). The Rheology of Blood Flow in Branched Arterial System. Applied rheology (Lappersdorf, Germany : Online) 15(6): 398-405.

Tan, F.P.P., Soloperto, G., Wood, N.B., Thom, S., Hughes, A. \& Xu, X.Y. (2008). Advanced Computational Models for Disturbed and Turbulent Flow in Stenosed Human Carotid Artery Bifurcation. IFMBE Proceedings (p. 390-394).

Trainini, J. (2004). Consenso de patología de la aorta. Revista Argentina de Cardiología 72(5), 387-40I.

Ubal, S., Campana, D.M., Rodríguez, C.F., Berli, M.E. \& Paolo, D. (20।0). Análisis Computacional de flujos sanguíneos utilizando modelos 3D axisimétricos y el método de elementos finitos (Vol. 6072). Entre Ríos, Argentina. Recuparado de http:// www.bioingenieria.edu.ar/grupos/biomecompu/Archivos de Proyectos/Rese_PID 6072_Analisis_computacional_flujos_ sangineos_axisimetricos.pdf.

Vasava, P., Jalali, P. \& Dabagh, M. (2009). Computational Study of Pulsatile Blood Flow in Aortic Arch: Effect of Blood Pressure. IFMBE Proceedings (Vol. 25, p. I I98-I20I).

Watson, B.T. (2007). A Fluid Structure Interaction Simulation of Blood Flow in the Left Heart. University of Oklahoma.

Zakaria, H., Robertson, A. \& Kerber, C. (2008). A Parametric Model for Studies of Flow in Arterial Bifurcations. Annals of biomedical Engineering 36(9): I515-1530. doi:।0.1007/ sl 0439-008-9531-y.

Zhao-rong, L., Gang, X., Yong, C., Zhong-zhao, T. \& Kai-rong, Q. (2003). An analysis model of pulsatile blood flow in arteries. Applied Mathematics and Mechanics 24(2): 230-240. doi: I 0.1007/BF02437630. 